\title{
Leveraging Partnerships to Maximize Global Health Security Improvements in Guinea, 2015-2019
}

Claire J. Standley, Pia D. M. MacDonald, Aurelia Attal-Juncqua, Alpha Mahmoud Barry, Ebi Celestin Bile, Doreen L. Collins, Salomon Corvil, Diallo Boubabar Ibrahima, Jennifer J. Hemingway-Foday, Rebecca Katz, Kathy J. Middleton, Eileen M. Reynolds, Erin M. Sorrell, Soumah Mohamed Lamine, Abdoulaye Wone, and Lise D. Martel

In response to the 2014-2016 West Africa Ebola virus disease (EVD) outbreak, a US congressional appropriation provided funds to the US Centers for Disease Control and Prevention (CDC) to support global health security capacity building in 17 partner countries, including Guinea. The 2014 funding enabled CDC to provide more than 300 deployments of personnel to Guinea during the Ebola response, establish a country office, and fund 11 implementing partners through cooperative agreements to support global health security engagement efforts in 4 core technical areas: workforce development, surveillance systems, laboratory systems, and emergency management. This article reflects on almost 4 years of collaboration between CDC and its implementing partners in Guinea during the Ebola outbreak response and the recovery period. We highlight examples of collaborative synergies between cooperative agreement partners and local Guinean partners and discuss the impact of these collaborations in strengthening the above 4 core capacities. Finally, we identify the key elements of the successful collaborations, including communication and information sharing as a core cooperative agreement activity, a flexible funding mechanism, and willingness to adapt to local needs. We hope these observations can serve as guidance for future endeavors seeking to establish strong and effective partnerships between government and nongovernment organizations providing technical and operational assistance.

Keywords: Global health security, Surveillance, Workforce development, Laboratory strengthening, Public health emergency management

Claire J. Standley, PhD, is Assistant Research Professor; Aurelia Attal-Juncqua, MSc, is Senior Research Associate; Rebecca Katz, PhD, is Professor and Director; and Erin M. Sorrell, PhD, is Assistant Research Professor; all at the Center for Global Health Science and Security, Georgetown University, Washington, DC. Pia D. M. MacDonald, PhD, is Senior Director; Jennifer J. Hemingway-Foday, $\mathrm{MPH}$, is a Research Epidemiologist; and Eileen M. Reynolds, MA, is Senior IT Project Manager; all at RTI International, Research Triangle Park, NC. Alpha Mahmoud Barry, DrPH, is Professor and Executive Director, University of Conakry and Santé Plus Organization, Conakry, Guinea. Ebi Celestin Bile, MSc, is Team Lead, IDDS Project, IDDS West Africa-Guinea, FHI360, Conakry, Guinea. Doreen L. Collins, MPH, is Director, Global Project Management, FHI Clinical, Durham, NC. Salomon Corvil, MD, is FETP-Guinea Resident Advisor, AFENET, Conakry, Guinea. Diallo Boubabar Ibrahima, MPH, is Chief of Party, RTI International, Conakry, Guinea. Kathy J. Middleton, MPH, is a Public Health Advisor, and Lise D. Martel, PhD, is Guinea Country Director; both with the Division of Global Health Protection, Center for Global Health, the US Centers for Disease Control and Prevention, Atlanta, GA. Soumah Mohamed Lamine, MD, is an Emergency Management Technical Assistant, and Abdoulaye Wone, MD, is Public Health Coordinator; both with the International Organization for Migration, Conakry, Guinea. The findings and conclusions in this report are those of the authors and do not necessarily represent the official position of the US Centers for Disease Control and Prevention. 
$\mathbf{I}_{\mathrm{n}}^{\mathrm{N}}$ N March 2014, THE Guinea Ministry of Health formally declared what would become the 2014-2016 West Africa Ebola virus disease (EVD) outbreak. ${ }^{1}$ Individuals with EVD were spread from Guinea to Sierra Leone and Liberia. By June 2014, Médecins Sans Frontières (MSF) described the outbreak as "out of control." "In August, then World Health Organization (WHO) Director-General Margaret Chan declared the outbreak a public health emergency of international concern. ${ }^{3}$ In late 2014, the US Congress passed a $\$ 5.4$ billion appropriation package for both domestic and international actions to support EVD response efforts. ${ }^{4}$ Of this amount, the US Centers for Disease Control and Prevention (CDC) received $\$ 1.77$ billion, with $\$ 603$ million dedicated to international response activities and a further $\$ 597$ million to global health security capacity building in 17 partner countries, including Guinea, Sierra Leone, and Liberia, the 3 countries most affected by EVD. 5

Prior to the 2014-2016 West Africa Ebola outbreak, CDC's presence in Guinea consisted of 1 staff position seconded to the US Agency for International Development (USAID) to support the president's malaria initiative. The 2014 congressional funding made it possible for CDC to provide more than 300 deployments of personnel to Guinea during the Ebola response, establish a country office, and fund implementing partners through cooperative agreements to support global health security engagement efforts.

In February 2015, funds were awarded to 4 organizations to support efforts in Guinea: RTI International (RTI), George Washington/Georgetown University (GU), Agence de Médecine Préventive, and PCI Media Impact, Inc. In 2016, additional organizations, including WHO, the Guinea Ministry of Health, the African Field Epidemiology Network (AFENET), the International Medical Corps (IMC), and the International Organization for Migration (IOM), also received funding. In addition to supporting response efforts through the end of the outbreak in June $2016,{ }^{6}$ these partners focused on ensuring the long-term strengthening of 4 core capacities of the Guinean health system-workforce development, surveillance, laboratory, and emergency management-and preventing future epidemic-prone disease outbreaks.

This article summarizes almost 4 years of collaboration between CDC and its implementing partners in Guinea during the EVD response and the recovery period. We highlight examples of collaborative synergies between cooperative agreement partners and local Guinean partners, discuss the impact of these collaborations on successful outcomes, and describe how partnership can benefit global health security implementation.

\section{Workforce Development}

The Field Epidemiology Training Program (FETP) trains individuals to conduct field investigations and collect, manage, analyze, and share surveillance data to facilitate science-based decision making. The FETP has 3 levels: frontline, intermediate, and advanced. ${ }^{7}$ Two of the 3 levels are offered in Guinea: FETP-Frontline and FETPIntermediate.

The FETP-Frontline 3-month program is designed to train district-level government staff who are likely to be the first to investigate a case or outbreak of infectious disease. The FETP-Intermediate 9-month program targets public health staff expected to train and supervise FETP-Frontline trainees and serves as a pool of candidates for the Advanced program. An FETP-Advanced regional 2-year program is offered in Burkina Faso. FETP graduates from the FETPIntermediate and Advanced levels fulfill the workforce development core capacity objectives under the International Health Regulations (IHR) (2005), the Global Health Security Agenda, and the Joint External Evaluation (JEE). ${ }^{7-9}$

During the EVD response, Guinea benefited from the assistance of FETP-Advanced graduates from the Democratic Republic of the Congo (DRC) ( 49 graduates), Haiti (2 graduates), and Cameroon (2 graduates). The performance of these FETP graduates facilitated the launch of the FETP in Guinea. In 2017, AFENET began to implement FETP-Frontline with assistance from RTI. AFENET had extensive expertise in implementing FETP in other African countries, ${ }^{10}$ but no previous experience in Guinea. RTI had been operating in Guinea since 2007. RTI's strong administrative team could quickly and effectively plan training and coordinate field activities and had a well-developed relationship with the ministry of health, but they lacked experience in implementing FETP.

AFENET recruited a francophone FETP graduate resident advisor who provided strategic and technical leadership, and it guided the ministry of health leadership through the sustainable and permanent institutionalization of the program. RTI provided logistical and administrative support for mentor training and field activities and hired a francophone epidemiologist from the DRC to serve as a coach for the mentors. RTI leveraged its simultaneous involvement in community-based surveillance, the implementation of District Health Information Software Version 2 (DHIS 2), ${ }^{11}$ and FETP to reinforce all 3 programs. RTI facilitated the inclusion of a training module on the DHIS 2 platform and its use for the analysis of surveillance data into the FETP curriculum, and the use of Integrated Disease Surveillance and Response (IDSR) ${ }^{12}$ tools in FETP mentoring. CDC, AFENET, IOM, and RTI collaborated with the IHR National Focal Point of the ministry of health's National Agency for Health Security (ANSS* in French) to enhance cross-border surveillance between neighboring districts in Guinea and Côte d'Ivoire and encourage cross-border coordination and data sharing between FETP-Frontline graduates (Figure 1).

*Agence Nationale de Sécurité Sanitaire. 


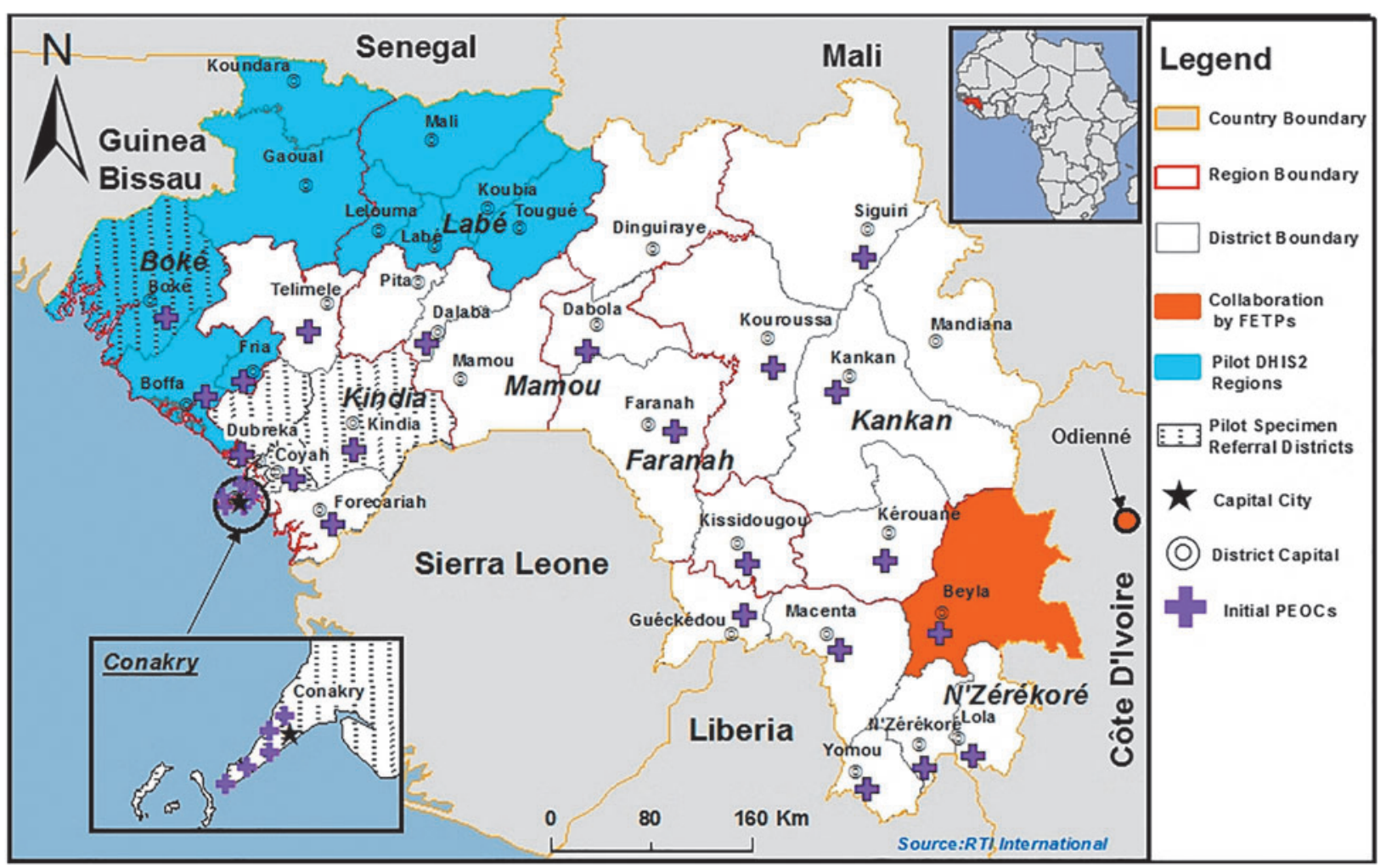

Figure 1. Map of Guinea, showing the locations of 4 of the collaborative activities described in the paper: the cross-border collaboration between Field Epidemiology Training Programs (FETPs) in Guinea and Côte d'Ivoire; the pilot regions for District Health Information Software (DHIS2) implementation; the pilot districts for the specimen referral projects; and the locations of each of the initial 28 subnational emergency operations center (PEOCs).

\section{Surveillance Systems}

The 2014-2016 West Africa EVD outbreak provided an opportunity to identify and address areas of improvement in the Guinean disease surveillance system. One priority was the development and countrywide implementation of standardized IDSR data collection tools for priority epidemic-prone diseases alongside a transition from paperbased reporting to a DHIS 2 electronic surveillance system.

The WHO's IDSR framework aims to strengthen districtlevel surveillance for priority diseases and translate surveillance and laboratory data into specific and timely response actions in accordance with the IHR (2005). ${ }^{12,13}$ In Guinea, the development and implementation of standardized IDSR data collection tools was a collaborative effort among RTI, CDC, the ministry of health, and WHO. Together, they formed a technical group and facilitated a series of workshops. Following the One Health approach, ${ }^{\dagger}$ per the "zoonotic disease" JEE technical area, the workshops included the Ministry of Health, the Ministry of Livestock, the

\footnotetext{
${ }^{\dagger}$ One Health is an approach to designing and implementing programs, policies, legislation, and research in which multiple sectors (human, animal, and environmental health) communicate and work together to achieve better public health outcomes.
}

Ministry of Environment, CDC, and surveillance and laboratory partners. Together, they reviewed and updated the country-specific notification forms for 13 priority diseases in Guinea. ${ }^{14}$ The IDSR tools include updated case notification forms (on paper and electronically in DHIS 2) and supportive job aids for health and laboratory workers at all levels of the system (national, regional, district, and community) to promote effective implementation of IDSR guidelines and collection of case data.

RTI worked with the ministry of health and partners to configure the case notification forms in the national DHIS 2 system using the Tracker application, which enables collection of individual-level data. Once entered into DHIS 2 at the district level, case data are immediately available to authorized users at all levels and can be analyzed using DHIS 2 data analysis tools or exported to other tools. The system enables the laboratories to register the receipt of laboratory samples for individual cases, input the results, and share them automatically via SMS or email. In late 2017, a pilot implementation using DHIS 2 for disease surveillance was successfully conducted in Boke and Labe administrative regions and then rolled out in the rest of the country (Figure 1).

The inclusion and participation of critical technical partners and of the ministries in the development and revision of the forms facilitated their subsequent approval and 
adoption from the national to the community level and their integration into the national DHIS 2 system. The multisectoral workshops also resulted in improved tools for zoonotic disease detection, reporting, and response. Three new zoonotic diseases - anthrax, brucellosis, and rabieswere added to the weekly surveillance system, and recommendations for enhancing surveillance of 2 zoonotic diseases already included in weekly surveillance-influenzalike illness and Ebola-were made.

In August 2018, CDC and its cooperative agreement partners joined with the ministries to conduct a training session for trainers on the new IDSR case notification forms, job aids, and the use of DHIS 2 to manage, report, analyze, interpret, and present the surveillance data. From December 2018 through March 2019, 55 trainers rolled out the comprehensive IDSR training to health workers at the regional, district, and community levels. Using a cascade approach, each level of the pyramidal structure of the ministry of health trained the next level. More than 1,200 health surveillance workers at the national, regional, and district levels across all of Guinea's 38 health districts were trained.

\section{LAbORatory Systems}

A tiered laboratory network with a robust referral and transport system of clinical specimens promotes early diagnostic testing to identify and/or confirm disease events and is a key JEE indicator under the "detect" pillar. The EVD outbreak provided an opportunity for cooperative agreement partners and the ministry of health to assess and identify areas needing improvement in the Guinean laboratory system. The development of a national specimen referral system, including a policy, standardized training, and operational tools for priority diseases, became an immediate priority.

CDC, GU, and IMC coordinated their support of the ministry of health to ensure a rapid and sustainable change in the specimen transport system. GU's primary role was to assist the ministry of health in developing a national specimen referral policy, while IMC focused on implementation. GU and IMC formalized their partnership through a memorandum of understanding (MOU), which helped to set clear expectations and define the roles and responsibilities of each cooperative agreement partner.

Under the leadership of the National Public Health Institute (INSP ${ }^{\S}$ in French), a technical working group was established to guide the development of the national specimen referral policy. The technical working group was composed of key individuals from the ministry of health, the faculty of medicine of the University Gamal Abdul Nasser of Conakry, and laboratory experts from CDC, GU,

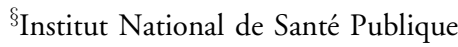

and IMC. It conducted a thorough review of the specimen referral and laboratory system in November 2015 through laboratory site visits across 4 national-level public health and veterinary laboratories.

Throughout 2016, under the leadership of INSP, the technical working group organized a series of laboratory partner workshops in Kindia, where drafts of the specimen referral policy were refined through small group discussions and a plenary session. The policy was presented to laboratory partners from the public health and veterinary sectors and validated by all through a consensus process before being officially handed over to the minister of health for final validation and signature in December $2016 .^{15}$

While GU assisted the ministry of health in developing its national specimen referral policy, IMC worked to ensure effective implementation of the new specimen referral system. From January to March 2016, IMC conducted a baseline survey focused on evaluating the existing specimen collection, testing, and transport capacity of community health centers and prefectural referral laboratories. The survey also determined the distance from the health centers and prefectural laboratories to the national reference laboratory in Conakry. Between February 2016 and January 2017, IMC implemented a pilot project to improve laboratory data collection and promote the implementation of the policy in the districts of Boké, Dubreka, Coyah, and Kindia and in Conakry (Figure 1). A mapping of health facilities was performed in these districts to select facilities qualified to serve as central hubs for the pilot specimen referral network and the peripheral facilities that would refer specimens to each respective referral laboratory, as described in the policy. The hub facilities received solar refrigerators for specimen storage, while peripheral facilities received specimen collection and packaging materials. IMC implemented a cost reimbursement scheme in each district to cover expenses incurred during specimen referral.

Finally, to strengthen laboratory technicians' capacities to safely and securely collect, package, store, and transport biological samples, IMC, GU, CDC, and the INSP developed training materials, standard operating procedures, and job aids and organized training sessions for health personnel and laboratory technicians. The roll-out of the standard operating procedures and job aids was done in coordination with the IDSR strengthening efforts led by the ministry of health, CDC, RTI, and WHO, to ensure close alignment between the surveillance and laboratory sectors for early and accurate disease detection.

\section{EMERgency Management}

Proper communication and coordination are critical capacities for public health emergencies and can be ensured through effective emergency management systems ${ }^{8,16,17}$ and the establishment of emergency operations centers (EOCs). ${ }^{18}$ During the EVD outbreak, CDC, the CDC Foundation 
(an independent nonprofit established by the US Congress to mobilize resources to support the work of the US CDC), and the Public Health Agency of Canada (PHAC) worked closely with the ministry of health to establish an emergency operations center at the national level. The CDC Foundation funded the renovation of a building to provide the needed infrastructure. Emergency management experts from CDC and PHAC staffed the national emergency operations center and mentored the ministry of health staff until the end of the Ebola response. The efficiency of the ministry of health emergency operations center in improving communication and coordination between partners prompted the establishment of a permanent national emergency operations center network. USAID's Office of Foreign Disaster Assistance funded IOM to renovate 28 district and communal facilities and transform them into public health EOCs (Figure 1).

In early 2015, CDC, GU, IOM, and PHAC collaborated to develop emergency management training materials for national and district ministry of health staff. GU led the development of training materials and documented training progress. IOM, in collaboration with CDC and PHAC, modified the curriculum according to the local field realities, and in 2016, with logistical and subject matter input from GU, trained 204 ministry of health staff in all districts. Representatives from regional and prefectural livestock and environmental departments were also included, in line with the One Health approach. IOM also facilitated a 1 -week study tour for 2 ministry of health emergency operations center staff to the Uganda National Public Health Emergency Operations Center. This experience gave them insights on emergency operations center operation and implementation. Two staff from ANSS and 2 from IOM also attended a 4-month training program at the CDC emergency operations center in Atlanta. IOM embedded their 2 trainees in the ministry of health emergency operations center to continue to assist the Guinean center development.

These capacity building efforts provided the ministry of health emergency operations center with a strong core staff to manage national and subnational emergency operations centers and continue to build capacity. In 2018, ministry of health emergency operations centers were activated 5 times - 3 times at the national level and twice at the district level_for yellow fever and measles outbreaks. To maintain capacity between outbreaks, the ministry of health, with support from partners including CDC, GU, and IOM, developed and performed a tabletop exercise at the national level and 3 more at the district level.

\section{ACHIEVEMENTS}

Guinea has come a long way since the Ebola outbreak of 2014-2016 in the areas of workforce development, surveillance systems, laboratory systems, and emergency management. Much of this success can be attributed to a collaborative spirit that was promoted by the government of Guinea and embraced by the CDC office in Guinea. A strong and transparent collaboration among the different cooperative agreement partners enabled each partner to contribute its individual strengths through 4 years of global health security capacity building. Figure 2 demonstrates the interconnectedness among all 4 technical areas and the collaboration among cooperative agreement partners.

A strong synergy developed among cooperative agreement partners during the development and implementation of the IDSR tools and their integration into the national DHIS 2 platform. In an effort to ensure that the tools were familiar to data managers and adapted to their needs, cooperative agreement partners collated and shared information from many parallel meetings and obtained full buy-in from different sectors of the ministry of health and other ministries involved before moving forward. This consensus process slowed down implementation but ensured sustainability and ownership by the national government.

Cooperative agreement partners treated other partners as potential extensions of their own capacity rather than as competitors. Training teams composed of staff from the different organizations allowed for a better use of the various partners' strengths. For example, RTI staff were able to strengthen both IDSR and DHIS 2 training with their data management, analysis, geographic information systems, and computer maintenance skills. The computer maintenance skills provided by RTI and the solar panels provided by WHO promoted greater longevity of the computers used for IDSR data collection. Moreover, the participation of implementing partners in meetings held to develop IDSR tools made it easier for all stakeholders to understand the importance and meaning of each field of information and provide quality training for implementation. Finally, the inclusion of DHIS 2 and of IDSR tools in the FETP curriculum allowed for additional opportunities to reinforce implementation of the tools. The integration of training on DHIS 2 and the new case notification forms in the recently launched University Gamal Abdul Nasser of Conakry master's of public health degree program also promotes longterm sustainability.

The spirit of collaboration facilitated the adoption of standardized IDSR data collection tools by surveillance and laboratory sectors of the ministry of health and ministry of livestock and was a model of successful implementation of a multisectoral One Health approach to IDSR. The IDSR tools provide a concrete example of how surveillance and laboratory staff of 2 ministries can benefit from a strong collaboration. The inclusion of laboratory information in IDSR tools made it easier to ensure that the data of the 2 sectors are linked and used for decision making.

Similarly, the integration of veterinarians and environmental health epidemiologists to FETP has already had a concrete impact on the response to zoonotic outbreaks. For example, in May 2018, a veterinary graduate of FETPFrontline provided the new IDSR priority disease list, 


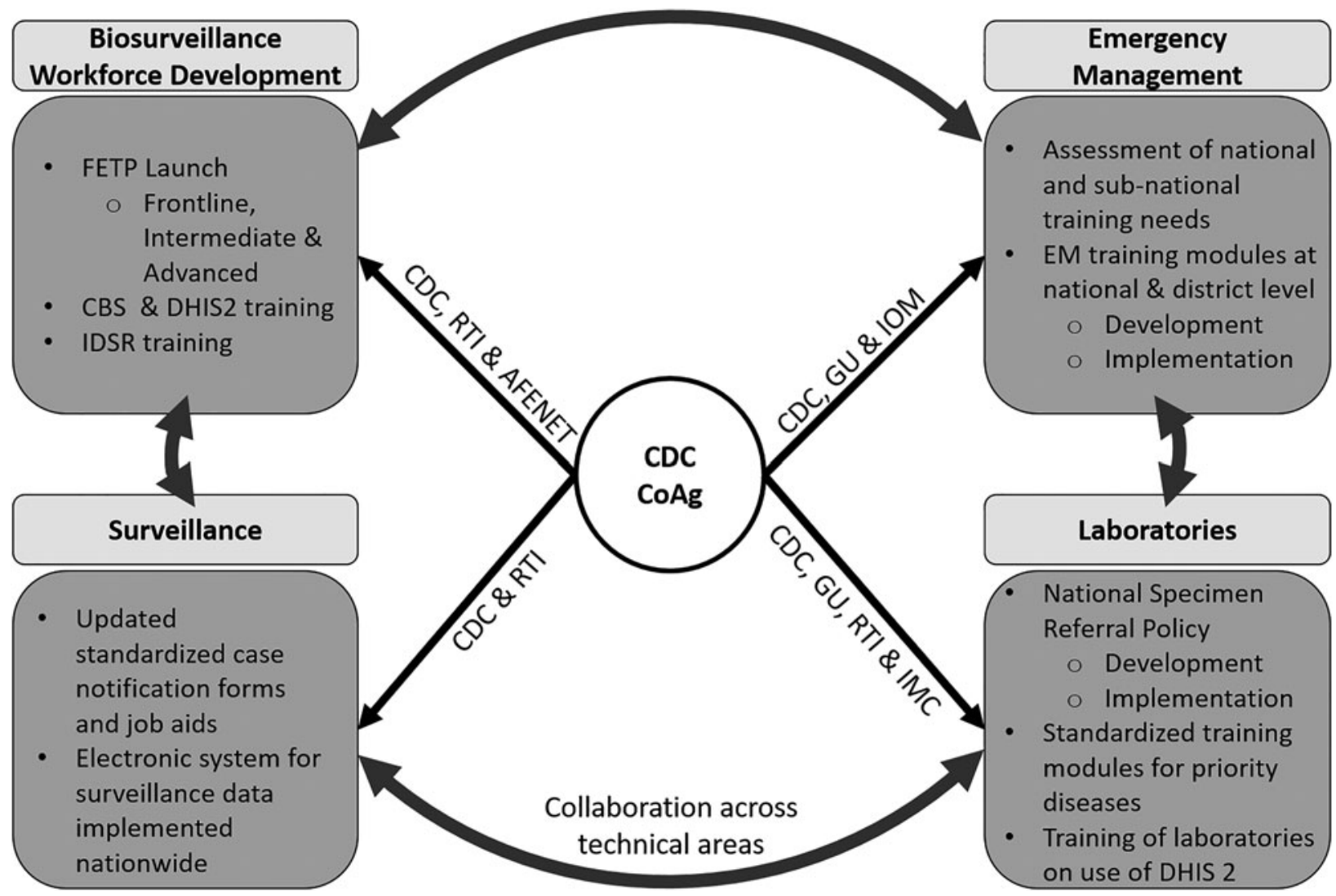

Figure 2. Schematic summarizing some of the activities conducted by CDC's cooperative agreement partners across the 4 primary technical areas of workforce development, surveillance systems, laboratory systems, and emergency management, and emphasizing coordination between partners and across technical areas for maximum impact. AFENET=African Field Epidemiology Network; $\mathrm{CBS}=$ community-based surveillance; $\mathrm{CDC}=\mathrm{US}$ Centers for Disease Control and Prevention; CoAg= Cooperative Agreement; DHIS $2=$ District Health Information Software Version 2; EM=emergency management; FETP = Field Epidemiology Training Program; $\mathrm{GU}=$ George Washington/Georgetown University; IDSR = Integrated Disease Surveillance and Response; IMC =International Medical Corps; IOM = International Organization for Migration; RTI = RTI International.

including zoonotic diseases, to all district livestock posts of Dubreka district. Two weeks later, using this information, the head of a livestock post in the district discovered and reported 4 cases of anthrax in local animals. The post had never previously reported a disease event. The ministries of health and livestock investigated these cases together and implemented control measures that avoided the consumption of contaminated animals, avoiding a potential outbreak of anthrax in people.

There are many ways to promote collaboration among partners. In Guinea, the ANSS facilitates collaboration as a core value by organizing regular meetings and vetting plans and activities through a consensus process that is inclusive of all partners. The ANSS holds a weekly epidemiologic meeting that is well attended by partners. It provides up-todate information and a valued opportunity for communication between partners. Similarly, the INSP's National Laboratory Committee holds monthly meetings with laboratory stakeholders.

In an effort to promote transparency and improved coordination across CDC cooperative agreement partners, RTI and GU initiated an annual "CDC Cooperative Agreement Partners Workshop," which was held at the end of each fiscal year. The workshop provided an opportunity for cooperative agreement partners to present to each other, CDC, and Guinean collaborators their proposed activities for the upcoming year. Through interactive small group discussions moderated by CDC, activities deemed as low priorities or duplicative by Guinean partners were removed or modified. The meeting provided an opportunity for cooperative agreement partners to display their strengths and achievements across the core capacities, allowed CDC to be transparent around their funding allocations, and made it possible for the government of Guinea to assert its leadership role in determining priority focus areas for the upcoming year.

\section{Challenges}

Working collaboratively, using consensus-based decision making, and involving relevant stakeholders can be time consuming, particularly in the context of a complex 
emergency like the 2014-2016 Ebola outbreak. However, these steps are imperative for building infrastructure that will survive beyond the initial international support and funding, as well as lasting relationships and trust.

In Guinea, collaboration between partners was complicated by the fact that it was initiated during the acute phase of the Ebola response. CDC and its cooperative agreement partners had to coordinate with each other but also take into account an already-crowded landscape of international assistance, including many actors whose expertise was geared specifically to the outbreak and humanitarian response as opposed to longer-term strengthening of health systems or building health security capacity. Officials from the ministry of health remarked to partners that it was difficult to keep track of all the activities, particularly given the high turnover of representatives from different international organizations. Likewise, the response needs were highly dynamic, with priorities changing rapidly as the outbreak progressed. The fluidity of the situation presented challenges for CDC and its cooperative agreement partners to stay aligned with government of Guinea priorities and ensure the correct counterparts were engaged with activities and plans, especially in the early months of the cooperative agreement awards, when the implementation teams for all 3 initial cooperative agreement partners were based outside of Guinea. At times, rapid adjustments to ensure coordination with the ministry of health, as the primary stakeholder for the global health security capacity-building activities, threatened to push coordination with other cooperative agreement partners into the background.

Internally, each cooperative agreement partner had to juggle its own procedural requirements and mission against the requests and needs of the ministry of health and CDC, particularly with respect to entering into collaborations. $\mathrm{CDC}$ and its cooperative agreement partners assumed the administrative burden of additional paperwork required to document the numerous changes that occurred during project implementation, such as redirection requests, establishing carry-over or no-cost extensions for funds when projects could not be implemented in time, and approvals for new activities or changes to existing work plans. These administrative requirements were compounded for activities where multiple cooperative agreement partners were involved.

Cooperative agreement partners had to work very closely with their CDC project officers and technical staff to ensure that their goals and work plans were closely aligned with country priorities but were still described in broad enough terms to allow for small changes to be accommodated when needed. In cases in which the proposed activities fell outside an implementer's area of expertise, the strong collaboration between cooperative agreement partners ensured each had access to sufficient technical, administrative, and logistical experience for successful implementation.

Finally, language was a significant barrier. The government of Guinea operates solely in French, with very few officials having any proficiency in English. In contrast, the headquarters of the majority of CDC's cooperative agreement partners are based in the United States and primarily function in English. There was no consistent approach to whether meetings and coordination calls should be held in French or English, with the decision largely dependent on who was present. The need to translate documents, especially technical documents such as work plans and journal articles, led to delays in implementation and quickly raised serious concerns about version control. At times, difficult decisions had to be made while balancing the need for partners' inclusion with speed of reaction. Cooperative agreement partners and the CDC office in Guinea mediated this problem by operating largely bilingual teams.

\section{LESSONS LEARNED}

The partnership model developed by CDC, cooperative agreement partners, and the ministry of health in Guinea provide numerous examples of how fostering a collaborative culture can lead to results that greatly transcend the sum of individual efforts. As these examples demonstrate, cooperative agreement partner collaboration can facilitate the adoption of creative and more effective models of implementation and encourage collaboration among different entities in the host government. Our experience in Guinea demonstrates the strong appreciation of the host government for proactive, transparent collaboration and information sharing among partners. It facilitated host government efforts to manage international assistance, ensured close alignment with national priorities, reduced duplication of effort, and maximized capacity building and sustainability.

Certain concrete mechanisms and actions can promote collaboration, especially in cases where a single funding agency is managing a larger number of implementing partners. From an administrative perspective, the multiyear cooperative agreement funding model was a key element for successful collaborations. Although new budgets were allocated each year, the reasonable expectation of renewed funding across multiple years facilitated the move from a competitive to a collaborative environment. Importantly, the ministry of health also had a cooperative agreement with CDC, which allowed the ministry of health to be empowered as an equal partner and understand the complexity of the administrative procedures and reporting requirements and the high standards of accountability held by each of the partners working under a cooperative agreement with CDC in Guinea.

The funder's management style and the degree to which they believe in the importance of coordination and collaboration play a key role in the development of a collaborative spirit. From the start, CDC ensured that the cooperative agreement structure provided sufficient flexibility, within the bounds of each partner's annual work 
plan, to adapt to the evolving needs of the government of Guinea. Moreover, CDC encouraged the inclusion of activities designed specifically to facilitate cooperation and collaboration, such as funding and personnel time for organizing the annual $\mathrm{CDC}$ and cooperative agreement partners' meeting. Other formal mechanisms, such as MOUs drafted between partners to help define each organization's responsibilities, were also encouraged. In addition, collaborative efforts and their resulting successes were regularly acknowledged by CDC staff on the ground. CDC set a tone of goodwill at the outset of the partnership by addressing problems in a transparent, consensus-driven, and solution-focused way.

The continuous presence on the ground of CDC's leadership, project officer, and technical staff for the full length of the cooperative agreement was invaluable for facilitating the administrative adaptability needed to implement project activities. CDC's Guinea country office's understanding of its implementing partners' budgets, missions, current work plans, expertise, and footprint in Guinea, and its strong relationship with critical decision makers at the ministry of health, made it possible to identify project opportunities with cooperative agreement partners, obtain permission for suggested activities, and afford cooperative agreement partners some leeway in implementation.

Regular and frequent opportunities were provided to cooperative agreement partners to interact with each other and learn about each other's missions, priorities, capabilities, and limitations, and openly discuss the possibility of collaboration. For example, CDC organized numerous meetings on important topics such as the development of the IDSR tools and the emergency management curriculum. Similarly, short meetings with the ministry of health partners were often defined around a specific topic rather than by organization, which allowed for the participation of all cooperative agreement partners involved. These direct interactions, combined with the annual meeting, made it easier for the ministry of health to be clear on the source of funding and the shared responsibilities of each cooperative agreement partner.

The annual cooperative agreement partners meeting proved to be a very successful mechanism for high-level coordination of activities not only between partners and with CDC, but also with the ministry of health. The meeting reassured the government of Guinea of our genuine interest in performing activities that support the mission and objectives of the ministry of health, our trust in and respect for its leadership, and our willingness to adjust and reprogram activities whenever logistically and legally possible. The approach created an atmosphere of goodwill where misunderstanding and mistakes were easily forgiven and the focus was on solutions rather than blame. It became a model to emulate. In 2018, the ANSS adopted this model to plan its yearly activities.

By building the cost and personnel time for coordination into partners' annual budgets and work plans, and by spreading the cost of coordination activities between CDC and cooperative agreement partners, the burden on any one organization was greatly reduced. The front-end cost of the meeting in time and money was greatly repaid through increased efficiency. It allowed for sharing of lessons learned, reduced redundancies and duplication of effort, fewer delays with project implementation through up-front ministry of health input, and a greater influence from a united voice. A better understanding of each cooperative agreement partner by the others also made it possible for each to serve as ears and eyes for the others, connect them with new partners, and remind the ministry of health of another partner's capacities in meetings where the partner might be absent. In all, it allowed for a greater and steady influence of all CDC cooperative agreement partners on the response. In an effort to facilitate the interactions and trust between cooperative agreement partners and the ministry of health, each cooperative agreement partner established a programmatic point of contact for the cooperative agreement whose responsibilities included regular coordination with government counterparts and cooperative agreement partners, and being the face of the agency in technical meetings.

Collaboration is a word that is often used loosely and sometimes means little more than participating in joint meetings. When collaboration becomes an expectation of operation in the public health sector, it allows for a new level of efficiency and effectiveness and possibly additional lives saved around the world.

\section{ReCOMMENDATIONS}

1. Regular communication and transparent information sharing from the government, funder, and across implementing partners is critical for successful collaborations.

2. Successful collaboration among implementing partners is facilitated by multi-year funding models that allow for flexibility in activities.

3. Implementing partners must be willing to adapt to local needs and reaffirm leadership of the local government.

4. The funder should have strong connections to the local government, ideally have an onsite presence, speak the local language, and be able to act as a conduit between the government and implementing partners.

5. Use MOUs or other written agreements to formalize partnerships and set clear expectations of roles and responsibilities among implementing partners.

6. Implementing partners should meet regularly (at least once a year) together with the funder and government officials to set priorities and identify pathways for collaboration and cooperation. 
7. The funder should encourage activities designed specifically to facilitate cooperation among implementing partners and with the government and allow funds to be allocated for this purpose.

\section{ACKNOWLedgMents}

The authors would like to thank the many individuals and organizations whose hard work, time, and expertise contributed to the success of the projects and activities described in this article. In particular, we would like to acknowledge the contributions of the Association of Public Health Laboratories, International Medical Corps, the Ministry of Health Bureau de Stratégie et Développement, and WHO. We also extend our deep gratitude to all the staff of the Cellule Nationale de Lutte contre Ebola/ANSS, and particularly the Director General, Dr. Sakoba Keita, for their trust and support throughout our work together in Guinea. This publication was supported by Cooperative Agreements U19GH001591 (RTI), U19GH001626 (GU), U19GH001876 (AFENET), and U19GH001761 (IOM), funded by the US Centers for Disease Control and Prevention.

\section{REFERENCES}

1. Centers for Disease Control and Prevention. 2014-2016 Ebola outbreak in West Africa. 2019. https://www.cdc.gov/ vhf/ebola/history/2014-2016-outbreak/index.html. Accessed December 14, 2019.

2. Médecins Sans Frontières. Ebola in West Africa: epidemic requires massive deployment of resources [news release]. June 21, 2014. http://www.msf.org/en/article/ebola-westafrica-epidemic-requires-massive-deployment-resources. Accessed November 19, 2019.

3. World Health Organization. Statement on the 1st meeting of the IHR Emergency Committee on the 2014 Ebola outbreak in West Africa. August 8, 2014. http://www.who.int/ mediacentre/news/statements/2014/ebola-20140808/en/. Accessed November 19, 2019.

4. Kates J, Michaud J, Wexler A, Valentine A. The U.S. response to Ebola: status of the FY2015 emergency Ebola appropriation. December 11, 2015. https://www.kff.org/ global-health-policy/issue-brief/the-u-s-response-to-ebolastatus-of-the-fy2015-emergency-ebola-appropriation/. Accessed November 19, 2019.

5. Fischer LS, Santibanez S, Jones G, Anderson B, Merlin T. How is CDC funded to respond to public health emergencies? Federal appropriations and budget execution process for non-financial experts. Health Secur 2017;15(3):307-311.
6. World Health Organization. Situation Report Ebola Virus Disease 10 June 2016. https://apps.who.int/iris/bitstream/ handle/10665/208883/ebolasitrep_10Jun2016_eng.pdf? sequence $=1$. Accessed January 7, 2020.

7. Jones DS, Dicker RC, Fontaine RE, et al. Building global epidemiology and response capacity with field epidemiology training programs. Emerg Infect Dis 2017;23(13):S158-S165.

8. World Health Organization. Joint External Evaluation Tool: International Health Regulations (2005). Geneva: WHO; 2016. http://apps.who.int/iris/bitstream/handle/10665/204368/ 9789241510172_eng.pdf;jsessionid=B3172A303E8D81C3CC DF42187CA20406? sequence=1. Accessed November 19, 2019.

9. André AM, Lopez A, Perkins S, et al. Frontline field epidemiology training programs as a strategy to improve disease surveillance and response. Emerg Infect Dis 2017;23(13).

10. Mukanga D, Tshimanga M, Wurapa F, et al. The genesis and evolution of the African Field Epidemiology Network. Pan Afr Med J 2011;10(Suppl 1):2

11. Health Information Systems Program. District Health Information Software Version 2 (DHIS 2). 2018. https://www. dhis2.org/. Accessed November 19, 2019.

12. Kasolo F, Yoti Z, Bakyaita N, et al. IDSR as a platform for implementing IHR in African countries. Biosecur Bioterror 2013;11(3):163-169.

13. World Health Organization, Centers for Disease Control and Prevention. Technical Guidelines for Integrated Disease Surveillance and Response in the African Region. 2d ed. 2010. http://www.afro.who.int/sites/default/files/2017-06/IDSRTechnical-Guidelines_Final_2010_0.pdf. Accessed November 19, 2019.

14. Ministère de la Santé. Plan de Renforcement de la Surveillance des Maladies à Potentiel Epidémique en Guinée (20152017). 2015.

15. Standley CJ, Muhayangabo R, Bah MS, et al. Creating a national specimen referral system in Guinea: lessons from initial development and implementation. Front Public Health 2019;7: 83.

16. Borchert JN, Tappero JW, Downing R, et al. Rapidly building global health security capacity-Uganda demonstration project, 2013. MMWR Morb Mortal Wkly Rep 2014;63(4): 73-76.

17. World Health Organization. Framework for a public health emergency operations centre. Geneva: WHO; 2015. http:// www.who.int/ihr/publications/9789241565134_eng/en/. Accessed November 19, 2019.

18. Frieden TR, Damon IK. Ebola in West Africa-CDC's role in epidemic detection, control, and prevention. Emerg Infect Dis 2015;21(11):1897-1905.

Address correspondence to: Claire J. Standley, PhD, MSc Assistant Research Professor Center for Global Health Science and Security Georgetown University Washington, $D C$

Email: claire.standley@georgetown.edu 